UMD-PP-94-50

September 1, 2021

\title{
Natural Doublet-Triplet Splitting in Supersymmetric SO(10) Models
}

\author{
Dae-Gyu Lee and R. N. Mohapatra \\ Department of Physics, University of Maryland \\ College Park, Maryland 20742
}

\begin{abstract}
We construct a supersymmetric $\mathrm{SO}(10)$ model, where the DimopoulousWilczek mechanism for doublet-triplet splitting is stable under the addition of Higgs superfields belonging to $\mathbf{1 2 6}+\overline{\mathbf{1 2 6}}$ needed to implement the see-saw mechanism for neutrino masses and where the charged fermion and neutrino mass spectra arise from a single set of $\mathbf{1 0}$ and $\overline{\mathbf{1 2 6}}$ Higgs representations.
\end{abstract}

\footnotetext{
${ }^{1}$ Work supported in part by a grant from the National Science Foundation
} 
In discussing supersymmetric grand unified theories, one has to deal with the vexing problem [囬] of doublet-triplet splitting. The problem arises from the simultaneous requirement that the $\mathrm{SU}(2)_{L}$ doublet and the color triplet submultiplets of a Higgs multiplet that generates fermion masses must have very disparate masses: the doublet mass must be of order of the electroweak scale $v_{w k}$ to cause $\mathrm{SU}(2)_{L} \times \mathrm{U}(1)_{Y}$ breaking whereas the color triplet mass must be of order of the GUT scale, $M_{U}$ in order to suppress rapid proton decay. The simplest way to achieve this is to fine tune the parameters of the superpotential. While such a tree level fine tuning is protected by quantum corrections, thanks to the non-renormalization theorem of supersymmetry, it requires unnatural adjustment of parameters and is unlikely to be the path chosen by nature. It is somehow more believable, if underlying group theoretical constraints guarantee the splitting for arbitrary choice of parameters of the theory. This possibility is called natural (or automatic) doublet-triplet splitting. In this letter, we study this question in the framework of realistic $\mathrm{SO}(10)$ models. Needless to say that recent indications for neutrino masses in various experiments have made $\mathrm{SO}(10)$ a more interesting GUT model to consider. It is therefore timely to address different aspects of these models.

In the minimal SO(10) models, Higgs superfields belonging to 10-dim. representations of $\mathrm{SO}(10)$ are used to generate bulk of the quark and lepton masses. One important prediction of the dominance of 10-dim. Higgs in generating fermion masses is the equality $m_{b}=m_{\tau}$ at GUT scale. When extrapolated to the weak scale, this relation predicts the ratio $m_{b} / m_{\tau}$ in good agreement with observations. The 10-dimensional multiplet contains two $\mathrm{SU}(2)_{L}$-doublets (which we denote by $\left.H_{u}, H_{d}\right)$ and a color triplet and anti-triplet (denoted here as $\xi_{1}(\mathbf{3})+\xi_{2}(\overline{\mathbf{3}})$ ). The problem of doublet-triplet splitting in $\mathrm{SO}(10)$ model boils down to understanding why $M_{\xi} \simeq M_{U}$ whereas $m_{H_{u, d}} \simeq v_{w k}$. Similar discussions will apply to the 126-dim. representation too, when it plays a role similar to the 10-dim. multiplet.

Several years ago, Dimopoulos and Wilczek [2] (DW) suggested a way to solve this problem for 10-dim. multiplet. They proposed using a 45-dim. Higgs multiplet (denoted by $\mathbf{A}$ ) to break the $\mathrm{SO}(10)$-symmetry by giving vev only to the $(1,1,15$ ) submultiplet of $\mathbf{A}$ under $\mathrm{SU}(2)_{L} \times \mathrm{SU}(2)_{R} \times \mathrm{SU}(4)_{C}$ subgroup and keeping the $(1,3,1)$ component to have zero vev naturally. Since $\mathbf{A}$ is anti-symmetric in the $\mathrm{SO}(10)$ indices, the implementation of this mechanism requires that there be at least two 10-Higgs representations (denoted by $H_{1}$ and $H_{2}$ ). It is then clear that, if

$$
<A>=\eta \otimes \operatorname{diag}(p, p, p, 0,0)
$$


where

$$
\eta \equiv\left(\begin{array}{cc}
0 & 1 \\
-1 & 0
\end{array}\right)
$$

a coupling in the superpotential of the form $\mathbf{A} H_{1} H_{2}$, will make all the triplets superheavy while leaving the four doublets $H_{1 u}, H_{1 d}, H_{2 u}$, and $H_{2 d}$ light . The subscripts $u$ and $d$ denote the doublets that can couple to up and down type quarks respectively. Since more than two light doublets are known to effect unification of couplings in an adverse manner, one will have to make one pair of these doublets superheavy. One can solve this problem (a) by letting one of the 10-Higgs multiplets (say, $H_{2}$ ) not couple to fermions and (b) by giving $H_{2}$ a direct superheavy mass in the superpotential, i.e., $\mu \mathrm{H}_{2} \mathrm{H}_{2}$. It has recently been argued [3] that this may not lead to a strong suppression of proton decay though enough to keep the model phenomenologically viable. We will not worry about this in order to keep the model simpler.

When one tries to implement the DW idea in realistic models, one immediately runs into difficulties. To appreciate this, let us first note that, a SUSY SO(10) model must have a $\mathbf{1 2 6}+\overline{\mathbf{1 2 6}}$ multiplet pair [4] in order to implement the see-saw mechanism to understand the small neutrino masses [5]. In the presence of the $\mathbf{1 2 6}+\overline{\mathbf{1 2 6}}$ pair (denoted by $\Delta+\bar{\Delta}$ ), one has the coupling $\Delta A \bar{\Delta}$, which induces a vev for the $(1,3,1)$ component of $\mathbf{A}$, thereby destroying the doublet-triplet splitting. If the coupling is forbidden and the B-L symmetry breaking by $\Delta+\bar{\Delta}$ vev's is accomplished by a term in the superpotential of the form $\left(\Delta \bar{\Delta}-M^{2}\right) S$ (S being a gauge singlet superfield), then there are a large number of light superfields (due to the SU(126) symmetry of the superpotential), that destroy the good unification properties. Something different must therefore be done.

A second requirement that we demand of the minimal $\mathrm{SO}(10)$ theory, is inspired by the recent observation [6] that a single $\mathbf{1 0}$ and single $\overline{\mathbf{1 2 6}}$ coupling to fermion generations can cure both the problem of bad mass relations for the second generation quarks and leptons, i.e., $\left(m_{s}=m_{\mu}\right.$ and $m_{d}=m_{e}$ at $\left.M_{U}\right)$ and also generate neutrino masses, provided the light Higgs doublets responsible for charged fermion masses is a linear combination of doublets from the $\mathbf{1 0}$ and $\overline{\mathbf{1 2 6}}$ multiplets. To achieve this mixing, the $\mathrm{SO}(10)$ symmetry must be broken by a $\mathbf{2 1 0}$-dim. Higgs multiplet and that there be no extra light doublets or color triplet fields left over from the $\Delta$ or $\bar{\Delta}$ in this process. It turns out that if the superpotential contains only a term of the form $\Phi \Delta H$ (and no $\Phi \bar{\Delta} H$ where $\Phi$ and $H$ respectively denote the 210 and 10 dimensional multiplets), this requirement is satisfied. Combinations of $\mathbf{4 5}+\mathbf{5 4}$ often used (e.g. in Ref. [3]) are not adequate for this purpose. This is a 
non-trivial constraint on model building. Below we present a model which satisfies all our requirements, i.e.,

(a) correct GUT symmetry breaking down to $\mathrm{SU}(3)_{C} \times \mathrm{U}(1)_{e m}$,

(b) only one pair of light Higgs doublets obtained naturally due to the existence of a symmetry (perhaps softly broken),

(c) the light doublets (both $H_{u}$ and $H_{d}$ types) are linear combinations of the doublets in the $\mathbf{1 0}$ and $\overline{\mathbf{1 2 6}}$ multiplets.

The Model: We consider the local symmetry group of the model to be $\mathrm{SO}(10)$, with an additional global symmetry $\mathrm{U}(1)_{P Q} \times Z_{16}^{H}$ with both symmetries softly broken by some dimension two terms in the superpotential.

We will demand the symmetry to be respected only by dimension three and higher terms of the superpotential and not by the soft dimension two terms. Thus, there is no light axion in the model. The following set of Higgs multiplets are chosen for the model: $\Phi_{1}(210), \Phi_{2}(210), A(45), \Delta(126), \bar{\Delta}(\overline{126}), H_{1,2}(10)$. Their transformation properties under the global symmetry groups are given in Table 1 . (We denote the sixteenth root of unity by $z$.)

The gauge invariant superpotential can be written as a sum of three terms:

$$
W=W_{m}+W_{H}^{(3)}+W_{H}^{(2)}
$$

where

$$
\begin{aligned}
W_{H}^{(3)} & =\lambda_{1} \Phi_{1}^{2} \Phi_{2}+\lambda_{2} \Phi_{2} \Delta \bar{\Delta}+\lambda_{3} \Phi_{1} A A+\lambda_{4} A H_{1} H_{2}+\lambda_{5} \Phi_{1} \Delta H_{1} \\
W_{H}^{(2)} & =\mu_{1} \Phi_{1}^{2}+\mu_{2} \Phi_{2}^{2}+\mu_{3} \Delta \bar{\Delta}+\mu_{4} A A+\mu_{5} H_{2} H_{2}+\mu_{6} \Phi_{1} \Phi_{2} \\
W_{m} & =h_{a b} \Psi_{a} \Psi_{b} H_{1}
\end{aligned}
$$

We choose all main parameters $\mu_{i}$ to be of order of the GUT scale $M_{U}$. The two terms $W_{H}^{(3)}+W_{H}^{(2)}$ lead to a number of degenerate supersymmetric minima, one of which has the desired pattern of symmetry breaking with $<A>$ given in Eq. (1) naturally with the vev of $\Phi_{1}$ only along the $(1,1,1)$ and $(1,1,15)$ directions and naturally with that of $\Phi_{2}$ along the $(1,1,1),(1,1,15)$, and $(1,3,15)$ directions. By the Dimopoulos-Wilczek mechanism, this leads to natural doublet-triplet splitting for both $H_{1}$ and $H_{2}$; the $\mu_{5}$ term makes one pair of the light doublets superheavy as required phenomenologically.

To show that this pattern of vev's preserves supersymmetry down to the electroweak scale, we call $\left\langle\Phi_{i}(1,1,1)\right\rangle=a_{i},\left\langle\Phi_{i}(1,1,15)\right\rangle=b_{i},\left\langle\Phi_{i}(1,3,15)\right\rangle=c_{i}$, $<A(1,1,15)\rangle=p,\langle A(1,3,1)\rangle=q,\langle\Delta(1,3, \overline{10})\rangle=\langle\bar{\Delta}(1,3,10)\rangle=v_{R}$. All these vev's are of order $M_{U}$. Let us first write down the vanishing F-term conditions 
for the $\langle A\rangle$; we get

$$
\begin{aligned}
& 2 \mu_{4} p+2 \lambda_{3} \frac{\sqrt{2}}{3} p b_{1}+2 \lambda_{3} \frac{1}{\sqrt{6}} q c_{1}=0 \\
& 2 \mu_{4} q+2 \lambda_{3} \frac{1}{\sqrt{6}} q a_{1}+2 \lambda_{3} \frac{1}{\sqrt{6}} p c_{1}=0 .
\end{aligned}
$$

From this, we first see that there is a solution of Eq. (6) for which $p \neq 0$ and $q=0$ if $c_{1}=0$. This is the vacuum we will focus on and see if the rest of the F-term conditions are satisfied for arbitrary values of the parameters in the superpotential. To see this, let us write down [7] those $F=0$ conditions with $c_{1}=0$ and $q=0$.

$$
\begin{aligned}
& 0=2 \mu_{1} a_{1}+\mu_{6} a_{2}, \\
& 0=2 \mu_{1} b_{1}+\mu_{6} b_{2}+2 \lambda_{1} \frac{b_{1} b_{2}}{9 \sqrt{2}}+\lambda_{3} \frac{\sqrt{2}}{3} p^{2}, \\
& 0=\mu_{6} c_{2}+2 \lambda_{1}\left(\frac{a_{1} c_{2}}{6 \sqrt{6}}+\frac{b_{1} c_{2}}{9 \sqrt{2}}\right) . \\
& 0=2 \mu_{2} a_{2}+\mu_{6} a_{1}+\lambda_{2} \frac{v_{R}^{2}}{10 \sqrt{6}}, \\
& 0=2 \mu_{2} b_{2}+\mu_{6} b_{1}+\lambda_{1} \frac{b_{1}^{2}}{9 \sqrt{2}}+\lambda_{2} \frac{v_{R}^{2}}{10 \sqrt{2}}, \\
& 0=2 \mu_{2} c_{2}+\lambda_{2} \frac{v_{R}^{2}}{10} . \\
& 0=2 \mu_{2} v_{R}+\lambda_{2}\left(\frac{a_{2} v_{R}}{10 \sqrt{6}}+\frac{b_{2} v_{R}}{10 \sqrt{2}}+\frac{c_{2} v_{R}}{10}\right) .
\end{aligned}
$$

Eqs. (7)-(9) arise from the F-terms corresponding to $\Phi_{1}, \Phi_{2}$, and $\Delta$ (or $\bar{\Delta}$ ), respectively. It is easy to see that this has non-trivial solutions for all the vacuum expectation values for arbitrary choice of the parameters of the model. This establishes the naturalness of the doublet-triplet splitting.

Let us now write down the doublet Higgsino mass matrix and isolate the massless pair of the Higgs doublets, that generate electroweak symmetry breaking. First we note that the doublets in $H_{2}$ pick up superheavy mass due to the $\mu_{5}$ term and are completely decoupled from the other doublets. Denoting the rest of the 
doublets by $\left(\Phi_{2 u}, \Phi_{1 u}, \Delta_{u}, \bar{\Delta}_{u}, H_{1 u}\right)$ and $\left(\Phi_{2 d}, \Phi_{1 d}, \bar{\Delta}_{d}, \Delta_{d}, H_{1 d}\right)$, we can write down their mass matrix in the basis where the above sets of fields denote the columns and rows respectively.

$$
\left(\begin{array}{ccccc}
\mu_{2} & \tilde{\mu}_{6} & 0 & \lambda_{2} v_{R} & 0 \\
\tilde{\mu}_{6} & \tilde{\mu}_{1} & 0 & 0 & \lambda_{5} v_{R} \\
0 & 0 & \tilde{\mu}_{3} & 0 & 0 \\
\lambda_{2} v_{R} & 0 & 0 & \tilde{\mu}_{3} & \lambda_{5} v_{U} \\
0 & 0 & \lambda_{5} v_{U} & 0 & 0
\end{array}\right)
$$

Analyzing this matrix, we conclude that it has two zero eigenstates given by:

$$
\begin{aligned}
H_{u} & =x_{1} H_{1 u}+x_{2} \bar{\Delta}_{u}+x_{3} \Phi_{1 u}+x_{4} \Phi_{2 u}, \\
H_{d} & =y_{1} H_{1 d}+y_{2} \bar{\Delta}_{d} .
\end{aligned}
$$

We therefore see that the light doublets have the desired property to solve the charged fermion mass puzzles of the simplest $\mathrm{SO}(10)$ models following Ref. [6]. All colored triplets in this model are superheavy - thus, proton decay is suppressed.

Let us now turn to the fermion sector. Note that $W_{m}$ in Eq. (5) has two deficiencies: (a) it does not give correct relation between $m_{s}$ and $m_{\mu}$, (b) the absence of the $\bar{\Delta}$ coupling makes the right-handed neutrinos massless. Both these problems are cured when we include the $Z_{16}^{H}$-invariant Planck scale induced dimension 4 terms in the superpotential, i.e.,

$$
W_{m}^{(1)}=\frac{1}{M_{P l}} f_{a b} \Psi_{a} \Psi_{b} \Phi \bar{\Delta} .
$$

A priori, there are three independent couplings, where the $\Psi \Psi$ bilinear transforms like 10, 120, 126; however, the fermion masses get contribution only from 10, $\overline{\mathbf{1 2 6}}$ type couplings. The 10 type couplings simply redefine the original 10 couplings, whereas the $\overline{\mathbf{1 2 6}}$ type couplings introduce the same structure to the fermion mass as in Ref. [6] and predictions of neutrino masses given in that reference carry over. If $f_{a b}$ are chosen to be order 1 to 3 and the $\bar{\Delta}$-vev's along the $(2,2,15)$ direction are chosen to be of order $100 \mathrm{GeV}$, the contribution of $W_{m}^{(1)}$ to fermion masses have the correct order of magnitude. Secondly, the right-handed neutrinos acquire a mass of order $\simeq 10^{12} \mathrm{GeV}$ or so if $v_{R} \simeq 10^{-1} v_{U}$. This scale for the right-handed neutrinos is helpful in understanding baryogenesis of the universe [9].

In summary, we have constructed an $\mathrm{SO}(10)$ model where the DimopoulosWilczek mechanism for doublet-triplet splitting is unaffected by the addition of 
$126+\overline{\mathbf{1 2 6}}$ multiplets needed for the see-saw mechanism for neutrino masses. We have also been able to show that once the lowest order Planck scale corrections are included, the light Higgs doublets have the correct structure to reproduce the desired quark-lepton mass spectra and lead to predictions for neutrino masses as in Ref. [6]. There are no undesirable light particles in the theory. We hope to have made clear the nontrivial nature of the constraints one would like to impose on a desirable $\mathrm{SO}(10)$ model and that by choosing appropriate symmetries, models satisfying all these constraints can indeed be constructed. Perhaps, the existence of one such model demonstrated in this letter will stimulate further thinking to construct simpler models that may yield further insight into $\mathrm{SO}(10)$ grandunified theories.

We thank K. S. Babu for some discussions.

\section{References}

[1] H. Georgi. Phys. Lett. 108B, 283 (1982); B. Grinstein. Nucl. Phys. B206, 387 (1982); A. Masiero, D. Nanopoulos, K. Tamvakis and Yanagida T. Phys. Lett. 115B, 380 (1982).

[2] S. Dimopoulos and F. Wilczek. NSF-ITP-82-07 (unpublished).

[3] K.S. Babu and S. Barr. Phys. Rev. D48 (1993) (to be published).

[4] C. S. Aulakh and R. N. Mohapatra. Phys. Rev. D28, 217 (1983); J. Malampi and J. Pulido. Nucl. Phys. B260, 391 (1985); J. Pulido. Nucl. Phys. B269, 119 (1986);

[5] M. Gell-Mann, P. Ramond, and R. Slansky. In Supergravity, edited by D. Freedman et. al. (North-Holland, Amsterdam, 1980); T. Yanagida. Proceedings of the KEK workshop, 1979 (unpublished); R. N. Mohapatra and G. Senjanovic. Phys. Rev. Lett. 44, 912 (1980).

[6] K. S. Babu and R. N. Mohapatra. Phys. Rev. Lett. 70, 2845 (1993).

[7] Xiao-Gang He and S. Meljanac. Phys. Rev. D41, 1620 (1990); Dae-Gyu Lee. University of Maryland preprint UMD-PP-94-49 (1993).

[8] G. Anderson, S. Dimopoulos, L. Hall, S. Raby and G. Starkman. LBL preprint LBL-33531 (1993).

[9] M. Fukugita and T. Yanagida. Phys. Lett. 174B, 45 (1986); P. Langacker, R. D. Peccei and T. Yanagida. Mod. Phys. Lett. A1, 541 (1986); M. Luty. Phys. Rev. D45, 455 (1992). 


\section{Table Caption}

Table 1: Global symmetry quantum numbers for different fields. $z=e^{i \pi / 8}$.

\begin{tabular}{|c|c|c|}
\hline \hline Supermultiplets & $\mathrm{U}(1)_{P Q}$ charge & $Z_{16}^{H}$ \\
\hline Matter multiplets & & \\
$\Psi_{3}$ & +1 & $z^{7}$ \\
$\Psi_{2}$ & +1 & $z^{7}$ \\
$\Psi_{1}$ & +1 & $z^{7}$ \\
\hline Higgs multiplets & & \\
$\Phi_{1}$ & 0 & $z^{12}$ \\
$\Phi_{2}$ & 0 & $z^{8}$ \\
$\mathrm{~A}$ & 0 & $z^{2}$ \\
$\Delta$ & +2 & $z^{2}$ \\
$\bar{\Delta}$ & -2 & $z^{6}$ \\
$H_{1}$ & -2 & $z^{2}$ \\
$H_{2}$ & +2 & $z^{12}$ \\
\hline \hline
\end{tabular}

Table 1 